\title{
A PORE-SCALE MODEL OF TWO-PHASE FLOW IN WATER-WET ROCK
}

\author{
DMITRIY SILIN AND TAD PATZEK
}

\author{
Lawrence Berkeley National Laboratory, Berkeley, California
}

\begin{abstract}
A finite-difference discretization of Stokes equations is used to simulate flow in the pore space of natural rocks. Numerical solutions are obtained using the method of artificial compressibility. In conjunction with Maximal Inscribed Spheres method, these computations produce relative permeability curves. The results of computations are in agreement with laboratory measurements.
\end{abstract}

\section{INTRODUCTION}

Flow properties of natural rocks or other porous media are eventually determined by the structure of the pore space. Core laboratory experiments are the main tool for studying these properties for scientific and practical applications. Recent progress in imaging and the growth of computing power make evaluation of rock properties from computer simulations a valuable complement to the traditional laboratory studies. Flow simulations on 3D images of the pore space provide valuable insights into the fundamental physics of Darcy flow. Comprehensive models and efficient computational algorithms make numerical studies a powerful tool with strong predictive capabilities. Various scenarios of fluid displacement in different wettability conditions can be tried on numerous images with very limited participation of the technical personnel. In some situations, as in the case of loosely consolidated formation, reliable measurements are practically impossible, and computer simulations can be the only available technique.

The literature presents a number of pore-scale studies of fluid flow. Fatt $[13,14,15]$ had pointed to the importance of cross-flow between the voids in the pore space for understanding Darcy's law. Instead of the model of a bundle of capillary tubes, which was routinely used before, Fatt proposed a pore-network model. He had demonstrated that a simple-geometry network of tubes is capable to capture the essential features of the flow in a porous medium and to provide valuable insights into the underlying microscopic mechanisms. However,

Key words and phrases. Pore-scale flow model, finite differences, creeping flow, Stokes equations, maximal inscribed spheres, relative permeability.

1 
a generic network does not necessarily reflect the pore space geometry of a particular rock. Oren with co-workers [5] have extended flow simulations to unstructured networks derived from three-dimensional tomography images of the pore space of natural rocks. The association of the network used in simulations with the specific rock data made possible to speak of "predictive capabilities" of the pore-scale modeling [30]. Numerous studies have been conducted to simulate two or multi-phase flows in mixed-wet rocks, to model fluid displacement hysteresis, etc. [8, 9, 31, 2].

The major computational advantage of the pore network approach is the simplified treatment of the flow equations. The flow in the entire network is presented as a sum of flows in individual channels, called pore throats. In the pore bodies, which are the junctions of multiple throats, the mass balance equations yield and analog of the Kirchhoff's first rule. The flow in each pore throat is described by a Poiseuille-type solution to the steady-state Navier-Stokes equations. This solution implies straightforward rules describing the conductivity of a flow channel with a relatively simple cross-section geometry [33, 32]. A more complex geometry may significantly complicate the description of the conductivity [4].

Despite all the simplifying assumptions, pore-network modeling has proved to be a useful tool providing insights into the physics and micromechanics of fluid flow in porous media. Comprehensive overviews of the pore-network approach over past five decades can be found in $[12,7]$. However, the problem of generating a network reflecting the specific properties of a given porous medium still remains a challenge. No universal routine procedure is available up until now. To bypass the challenges associated with extraction of a representative network, one can simulate fluid flow directly on the 3D image of the pore space. This approach includes the necessity to solve the flow equations on a $3 \mathrm{D}$ domain of an extremely complex geometry. One group of numerical simulations relies on the Lattice-Boltzmann method [25, 3, 29, 20]. This method utilizes the basic physics and is rather universal with respect to the range of phenomena suitable for numerical simulations. At the same time, a rigorous formulation of no-slip or fixed-pressure boundary conditions may be not as simple [28, 17]. Lattice-Boltzman simulations of two-phase flow with account for the interfacial phenomena require massive computations. The increment in the complexity between single-phase flow and two- or multi-phase flow is tremendous.

In this work, we employ a finite-difference approach to solving the Navier-Stokes equations. It makes possible incorporation of a wide variety of boundary conditions. The assumption is that the flow is 
steady and slow (creeping flow). Thus, the time derivative and the convective term in the flow equations can be dropped. At the microscopic scale of a computer-tomography image, the compressibility of the fluid can be neglected as well, which yields a linear system of Stokes equations. To simulate two-phase flow at a pore scale, we combine a finite-differences discretization of the Stokes equations with the Maximal Inscribed Spheres (MIS) algorithm [42, 38, 37]. MIS-calculations are based on simple rules end employ efficient cluster search algorithms [39], so that the simulations do not require super-computer power. The finite-difference approach to simulation of the fluid flow in the pore space has been in use for a long time [1, 36, 23, 24].

A digital image is a set of cubic voxels. Each voxel is labelled to distinguish between the void and the solid. The void voxels can be naturally used for a spacial discretization of the differential equations and boundary conditions. The large size of the computational domain, however, may complicate the flow simulations. For instance, a digital image of dimensions $100 \times 100 \times 100$ voxels is quite small from the point of view of representative high-resolution imaging. However, it requires solution of a boundary-value problem on hundreds of thousands of grid cells. The matrix of the discretized system is non-symmetric and the convergence can be quite slow. Even for Stokes creeping flow equations on a simple-geometry domain, a rigorous theoretical estimation of the convergence of the numerical solution to the exact one may be hardly possible. To figure out the computational parameters yielding reasonable accuracy of the simulations, we use test problems with known exact solutions.

Both the finite differences algorithms and the Lattice-Boltzmann method lead to numerical solutions to the Stokes equations. Apparently there is no obvious winner between the two approaches. Some finite-difference and Lattice-Boltzmann numerical schemes are very close to each other [19].

In the numerical simulations within this study, we employ the method of artificial compressibility developed by Chorin [10]. This method focuses on steady-state solutions, where the time-evolution of the flow is not of interest. We combine this approach with partitioning the entire digital image into parts. The partitioning helps to reduce the volume of computations to a degree that all the numerical examples presented below have been computed on a personal computer. The permeability of the entire sample is evaluated by averaging the permeabilities of the individual parts.

Prediction of the absolute permeability still remains uncertain due to the heterogeneity of the rock and, consequently, the high variability 
of the results. However, a combination of flow simulations with Maximal Inscribed Spheres calculations provides an opportunity to compute relative permeability curves. MIS-calculations proved to be an efficient way of generating realistic capillary pressure curves $[43,6]$. It turns out that the MIS-computed relative permeability curves are in a good agreement with the coreflood measurements.

The paper is organized as follows. In Section 2 we briefly overview the method of artificial compressibility of solving the steady-state Stokes equations. In Section 3 we describe the discretization and the numerical algorithm and verify the adequate convergence requirements. Section 4 presents a procedure of evaluation of the absolute and relative permeability from a tomography image by partitioning it into layers. Finally, Section 5 summarizes the findings and conclusions.

\section{The Method of ARTIFICIAL COMPRESSIBILITy}

In this section, we describe an implementation of the method of artificial compressibility [10]. Navier-Stokes equations for flow of a viscous incompressible fluid have the form

$$
\begin{aligned}
\frac{\partial}{\partial t} \mathbf{v}+(\mathbf{v} \cdot \nabla) \mathbf{v} & =-\frac{1}{\varrho} \nabla p+\nu \nabla^{2} \mathbf{v}+\mathcal{F} \\
\nabla \cdot \mathbf{v} & =0
\end{aligned}
$$

see [21]. Here $\mathbf{v}=\left(v_{x}, v_{y}, v_{z}\right)$ is the local velocity of the fluid flow, $p$ and $\varrho$ are the fluid pressure and density, $\nu$ is the coefficient of kinematic viscosity. We neglect the gravity or any other body forces, that is, $\mathcal{F}=0$.

In dimensionless variables:

$$
\mathbf{v}^{\prime}=\frac{\mathbf{v}}{V}, \quad \mathbf{r}^{\prime}=\frac{\mathbf{r}}{D}, \quad p^{\prime}=\frac{D p}{\varrho_{0} \nu V}, \quad t^{\prime}=\frac{\nu}{D^{2}} t
$$

where $V$ and $D$ are some characteristic velocity and distance scales, Equations (1) take on the form

$$
\frac{\partial}{\partial t^{\prime}} \mathbf{v}^{\prime}+\operatorname{Re}\left(\mathbf{v}^{\prime} \cdot \nabla\right) \mathbf{v}^{\prime}=-\nabla p^{\prime}+\nabla^{2} \mathbf{v}^{\prime}
$$

Here

$$
\operatorname{Re}=\frac{V D}{\nu}
$$

is the Reynolds number. Below, we will skip the prime in order to

avoid cumbersome notations. The idea of the artificial compressibility 
method is as follows: we replace the Navier-Stokes equations for an incompressible flow with a system of equations

$$
\begin{aligned}
\frac{\partial}{\partial t} \mathbf{v}+\operatorname{Re}(\mathbf{v} \cdot \nabla) \mathbf{v} & =-\nabla p+\nabla^{2} \mathbf{v} \\
\frac{\partial \rho}{\partial t}+\nabla \cdot \mathbf{v} & =0
\end{aligned}
$$

The dimensionless density $\rho$ is different from the physical fluid density $\varrho_{0}$ and is artificially introduced for the sake of computations. A linear relationship between the dimensionless pressure and the artificial density is assumed:

$$
\rho=\delta p
$$

Here $\delta$ is the artificial compressibility factor. Its value is not a physical property of the fluid, but a parameter to be chosen to facilitate the computations. If a solution to equations (6)-(7) converges to a steady solution as $t \rightarrow \infty$, then the time derivatives, including the one in Equation (7), vanish. In the limit, one obtains a steady-state solution for incompressible flow. Note that the magnitudes of the time derivatives actually evaluate the discrepancy of approximation to the steady state problem.

Our objective is steady-state creeping flow, where Re $\ll 1$ and the time derivatives vanish. Thus, we simplify the problem by putting $\operatorname{Re}=0$. This assumption eliminates the only nonlinear term in the equations $(6)-(7)$.

The flow equations must be complemented by appropriate boundary conditions. The computational domain comes from a digital image, which in our case is a subset of a rectangular box. We choose two opposite faces of this box and declare them the inlet and outlet boundaries. At these boundaries, we impose Dirichlet conditions for the pressures, zero-flow conditions for the lateral components of the flow velocities, and a zero Neumann condition for the component of velocity in the direction of the flow. The pressure enters the equations only through its derivatives. Therefore, it can be determined only up to a constant additive term. The Dirichlet conditions for the pressures eliminate the ambiguity in the numerical solution. The zero Neumann conditions for the velocities provide for the compatibility with Equation (7). Manwart et al. [23] used similar boundary conditions in a projection method of solving the Stokes equations [11].

\section{Discretization of The Differential EQUATIONS}

A digital image from a computer tomography scanner is a threedimensional array of cubic voxels. The size of each voxel is determined 


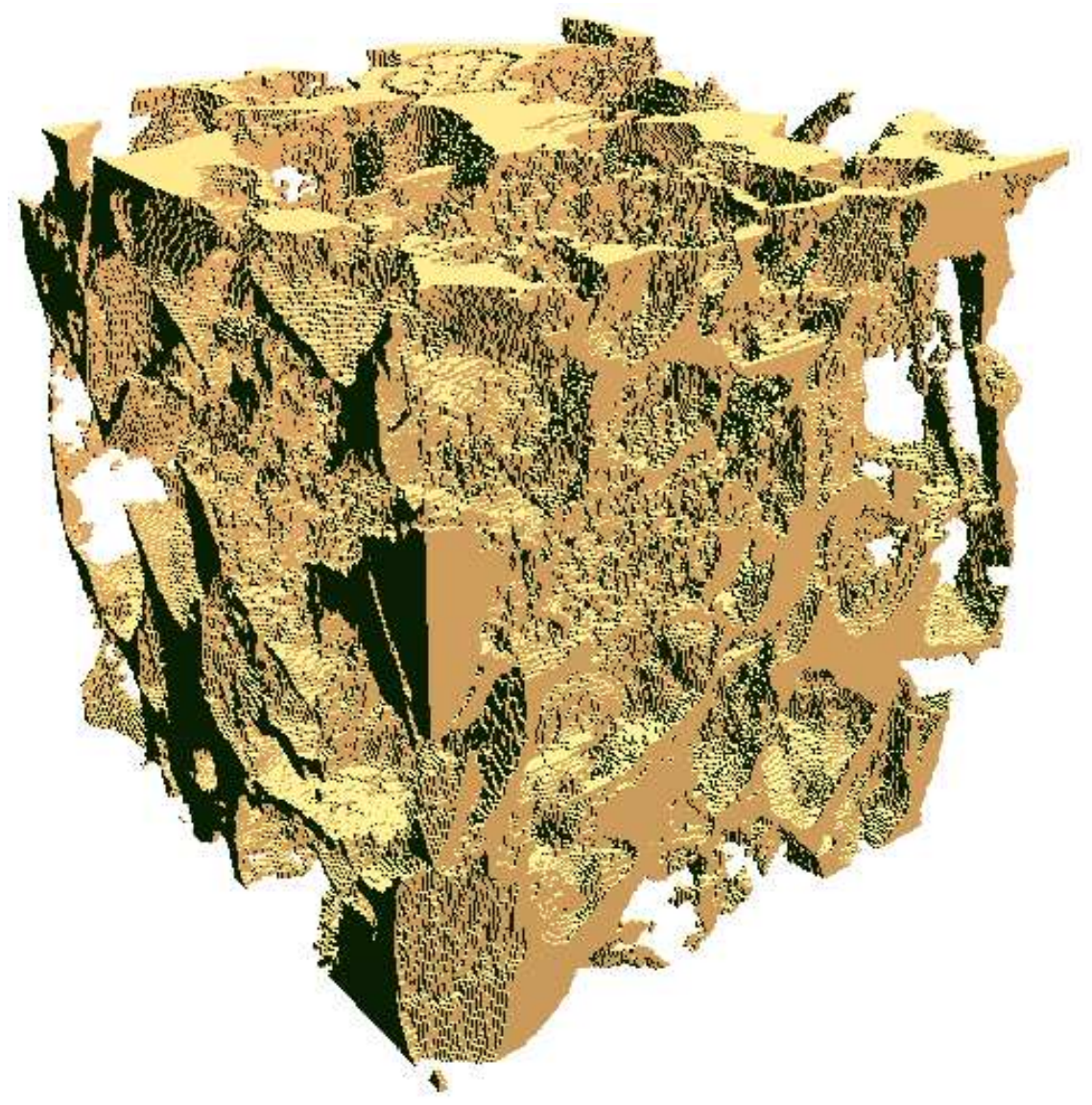

Figure 1. A 3D digital image of the pore space of sandstone, $250 \times 250 \times 250$ voxels.

by the resolution of the image. Some voxels represent the pore space, and some represent the solid. The pore space voxels naturally provide a grid for discretization. Figure 1 shows the digital image of the pore space of a sandstone.

To discretize equations (6)-(7) we employ a DuFort-Frankel pattern. We apply a staggered discretization scheme, also known as the markerand-cell method (MAC) [18].

The DuFort-Frankel pattern is an explicit three-layer scheme. As an explicit scheme, it requires relatively small amount of computations. At the same time, it is known to be unconditionally stable for some classes of boundary-value problems [35]. The convergence and stability of this scheme for Navier-Stokes equations have been confirmed by the numerical evidence [10]. 


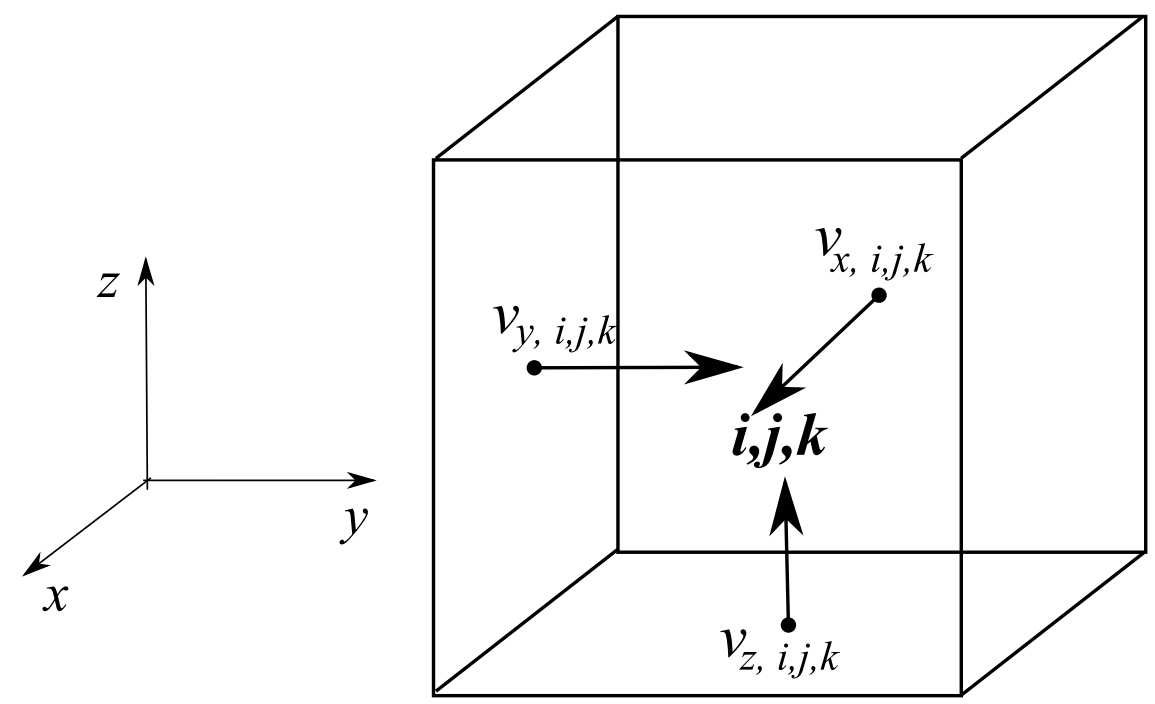

FiguRE 2. Voxel and fluid velocity components enumeration: $i_{v}=i+1, j_{v}=j+1$, and $k_{v}=k+1$, where $i, j, k$ are the indices of the voxel center.

The artificial compressibility factor $\delta$ should be selected to optimize the convergence and stability of the iterations. A smaller compressibility should lead faster to an incompressible flow solution, but may increase the risk of loosing stability by making the system of equations stiff. A larger compressibility can slow down the convergence to a steady solution, especially for Equation (7). We have used test computations to selected a suitable value for $\delta$.

In a MAC discretization, the velocities are evaluated at the center points of the interfaces between the grid cells, whereas the densities and pressures are evaluated at the volumetric centers of the voxels, Figure 2. Only the normal components of the velocities at the cell walls are used. For example, $v_{x}$ is evaluated only at the centers of those voxel faces, which are orthogonal to the $x$ coordinate axis.

3.1. Indexing convention. Let $N_{x}, N_{y}$, and $N_{z}$ be the dimensions of the grid. We enumerate the grid cells by zero-based indices $i=$ $0,1, \ldots, N_{x}-1, j=0,1, \ldots, N_{y}-1$, and $k=0,1, \ldots, N_{z}-1$. At each voxel, every velocity component is evaluated at the centers of two opposite walls. For the enumeration, we adopt the convention that the velocity is always associated with the upstream (with respect to the indexing) boundary. For example, Figure 2 shows the velocity components $v_{x, i, j, k}, v_{y, i, j, k}$, and $v_{z, i, j, k}$ associated by this indexing with the voxel $(i, j, k)$. Let $h$ denote the voxel edge length, and let the origin 
of the reference coordinate system be associated with the upstream corner of the entire image. Then the coordinates of the voxel center are $x=(i+0.5) h, y=(j+0.5) h$, and $z=(k+0.5) h$. The $x$ velocity component, $v_{x}$, is evaluated at the point with coordinates $(i h,(j+0.5) h,(k+0.5) h)$. The indexing conventions for other velocity components are similar. Figure 3 shows an example of indexation of the pressures and velocities at the cross-section of the interior part of the grid by the plane orthogonal to $z$ and passing through $z=(k+0.5) h$. The diamond markers label the points where the velocities are evaluated at the voxel walls. The circles mark voxel centers.

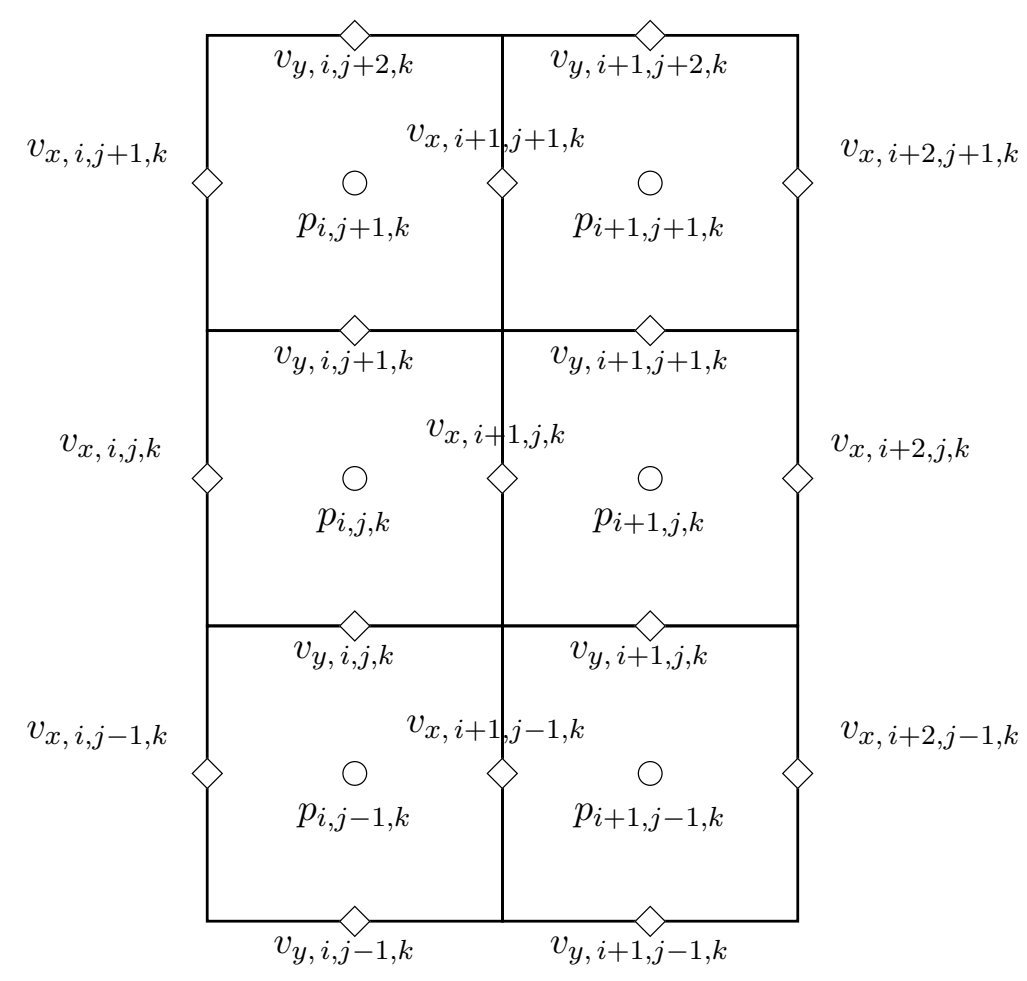

FiguRE 3. The circles mark the voxel centers, where the pressures are evaluated. The diamonds mark the centers of the interfaces between voxels, where the velocities are evaluated. This is a $2 \mathrm{D}$ slice of a $3 \mathrm{D}$ grid in plane $x, y$.

To write down the finite-difference equations, the velocities must be additionally evaluated at the outer downstream boundaries of the 
image, which are not covered by the indexing described above. Therefore, the regular part of the discrete domain described above is complemented by three two-dimensional velocity arrays associated with the ghost voxels.

3.2. Finite-difference equations. Let $\tau$ denote an increment step of the variable $t$. Note that the "time" variable $t$ in Equations (6)(7) is an iterations parameter, rather than a physically meaningful measure of time. A zero-based superscript index ${ }^{n}$ will denote the enumeration corresponding to $t$. For example, $v_{x, i, j, k}^{n}$ denotes the $n$ th iteration of the $x$-component of the velocity $\mathbf{v}$ evaluated at the center of the upstream (in direction $x$ ) wall of the voxel $i, j, k$. The discretized dimensionless pressures and artificial densities are denoted, respectively, by $p_{i, j, k}^{n}$ and $\rho_{i, j, k}^{n}$.

The DuFort-Frankel pattern is a three-layer scheme. Let the $(n-1)$ th and $n$-th iterations have been evaluated. Let us describe the procedure of calculatin of the $(n+1)$-th iteration for the velocities, artificial densities and pressures. The central difference provides a second-order approximation for the first-order derivative. For example,

$$
\begin{aligned}
& \left.\frac{\partial v_{x}}{\partial t}\right|_{i, j, k} ^{n}=\frac{v_{x, i, j, k}^{n+1}-v_{x, i, j, k}^{n-1}}{2 \tau}+O\left(\tau^{2}\right) \\
& \left.\frac{\partial p}{\partial x}\right|_{i, j, k} ^{n}=\frac{p_{i+1, j, k}^{n}-p_{i, j, k}^{n}}{h}+O\left(h^{2}\right)
\end{aligned}
$$

Although the pressures and velocities are evaluated at different points, the estimates in Equations (9) and (10) are valid at the same location: $x=i h, y=(j+0.5) h$, and $z=(k+0.5) h$. The second-order partial derivatives in $x, y$ and $z$ are approximated in the following way:

$$
\begin{aligned}
& \left.\frac{\partial^{2} v_{x}}{\partial x^{2}}\right|_{i, j, k} \approx \frac{\left(v_{x, i+1, j, k}^{n}-v_{x, i, j, k}^{n+1}\right)-\left(v_{x, i, j, k}^{n-1}-v_{x, i-1, j, k}^{n}\right)}{h^{2}} \\
& \left.\frac{\partial^{2} v_{x}}{\partial y^{2}}\right|_{i, j, k} \approx \frac{\left(v_{x, i, j+1, k}^{n}-v_{x, i, j, k}^{n+1}\right)-\left(v_{x, i, j, k}^{n-1}-v_{x, i, j-1, k}^{n}\right)}{h^{2}} \\
& \left.\frac{\partial^{2} v_{x}}{\partial z^{2}}\right|_{i, j, k} \approx \frac{\left(v_{x, i, j, k+1}^{n}-v_{x, i, j, k}^{n+1}\right)-\left(v_{x, i, j, k}^{n-1}-v_{x, i, j, k-1}^{n}\right)}{h^{2}}
\end{aligned}
$$

Equations (9)-(13) yield the following finite-difference approximation of the $x$ component of the creeping-flow approximation $(\operatorname{Re}=0)$ of 
Equation (6):

$$
\begin{array}{r}
\frac{v_{x, i, j, k}^{n+1}-v_{x, i, j, k}^{n-1}}{2 \tau}=\frac{v_{x, i-1, j, k}^{n}+v_{x, i+1, j, k}^{n}-v_{x, i, j, k}^{n-1}-v_{x, i, j, k}^{n+1}}{h^{2}} \\
+\frac{v_{x, i, j-1, k}^{n}+v_{x, i, j+1, k}^{n}-v_{x, i, j, k}^{n-1}-v_{x, i, j, k}^{n+1}}{h^{2}} \\
+\frac{v_{x, i, j, k-1}^{n}+v_{x, i, j, k+1}^{n}-v_{x, i, j, k}^{n-1}-v_{x, i, j, k}^{n+1}}{h^{2}} \\
+\frac{\rho_{i+1, j, k}^{n}-\rho_{i, j, k}^{n}}{\delta h}
\end{array}
$$

The $y$ and $z$ components of the Stokes equations are approximated in a similar manner. For the artificial compressibility equation (7), one obtains:

$$
\begin{array}{r}
\frac{\rho_{x, i, j, k}^{n+1}-\rho_{x, i, j, k}^{n-1}}{2 \tau}=\frac{v_{x, i, j, k}^{n}-v_{x, i-1, j, k}^{n}}{h}+\frac{v_{y, i, j, k}^{n}-v_{y, i, j-1, k}^{n}}{h} \\
+\frac{v_{z, i, j, k}^{n}-v_{z, i, j, k-1}^{n}}{h}
\end{array}
$$

The finite-difference Equations (14)-(15) provide second-order approximations to the respective partial differential equations.

3.3. Discretization of the boundary conditions. The problem under consideration involves two types of boundary conditions. Inside the sample image, at the pore walls, we impose no-slip conditions. At the boundaries of the whole image, the boundary conditions are different depending on the direction of the flow. We impose Dirichlet conditions for the pressures at the inlet and outlet faces, zero Dirichlet conditions for the lateral components of velocity, and a zero Neumann condition for the velocity component aligned with the macroscopic pressure gradient. For the velocities at the lateral boundaries of the domain, we impose zero Dirichlet conditions. In this section, we discuss the implementation of these boundary conditions in more detail.

3.3.1. Discretization of the no-slip boundary conditions at the pore walls inside the image. Incorporation of the no-slip boundary condition merely means an appropriate modification of Equations (14) when one of the nodes of the stencil occurs at a pore wall or inside the solid phase. There are several typical configurations, which can be considered individually. In the two-dimensional illustrations, the solid voxels are shaded and the pore voxels are blank. A solid diamond marker labels the center of the stencil of the discretized Stokes equation (14). The circles are voxel centers, which are simultaneously stencil center points 
for Equation (15). The stencils, on which the Stokes equations are discretized, are centered at the interfaces between pore voxels.

Handling a situation where the velocity component is orthogonal to the pore wall is straightforward. For example, in a configuration shown in Figure 4, the no-slip boundary condition, $v_{x, i, j_{v}, k}^{n}=0$, implies

$$
\left.\frac{\partial^{2} v_{x}}{\partial x^{2}}\right|_{i, j, k} ^{n} \approx \frac{v_{x, i+1, j, k}^{n}-v_{x, i, j, k}^{n+1}-v_{x, i, j, k}^{n-1}}{h^{2}}
$$

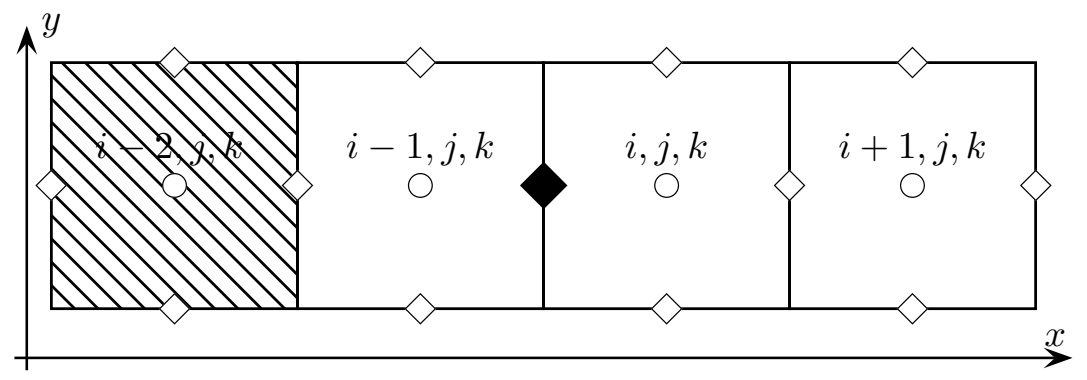

Figure 4. A stencil encountering a pore wall. The filled diamond marks the center of the stencil.

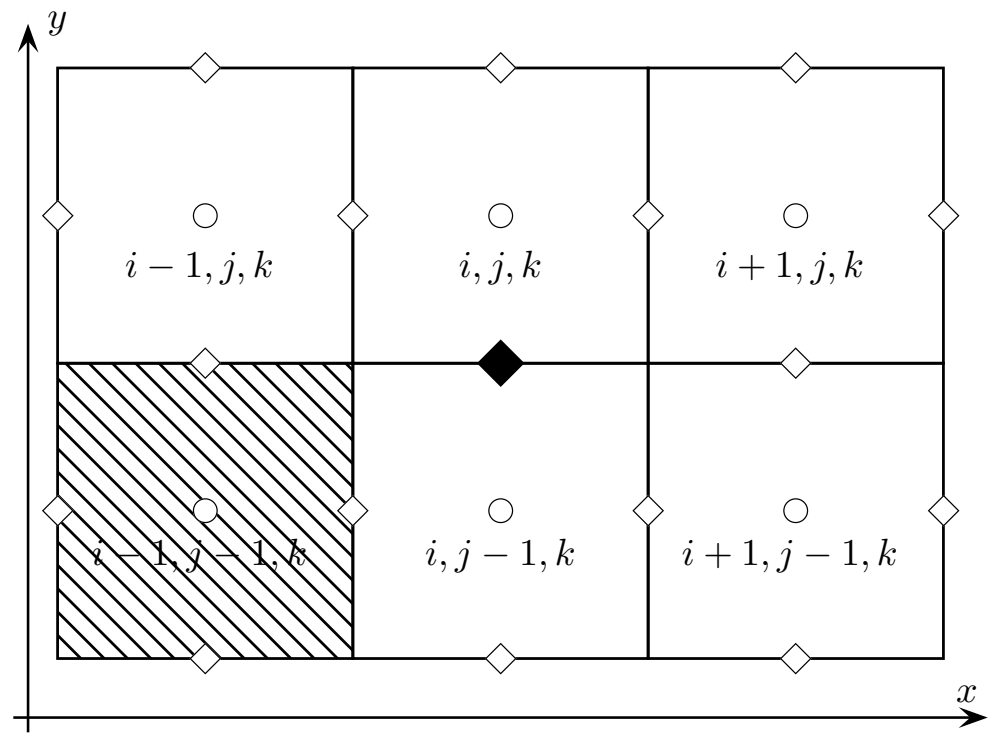

Figure 5. A stencil with a pore wall corner.

To show how to incorporate the no-slip boundary conditions into discretization of second partial derivative of a velocity component in an 


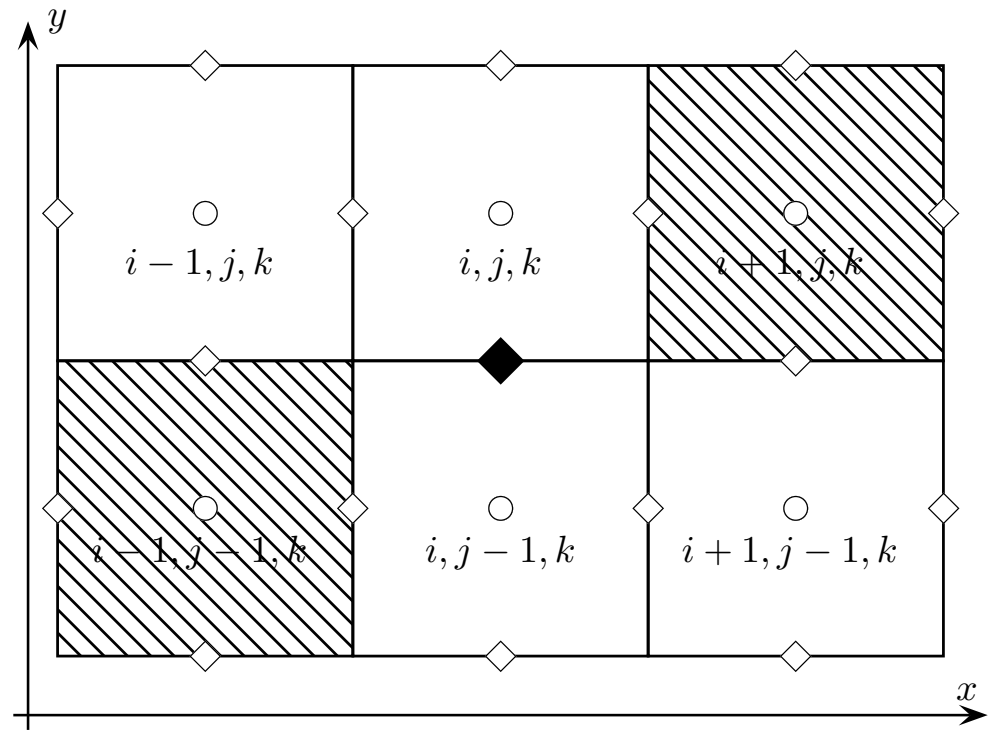

Figure 6. A stencil with two pore wall corners.

orthogonal direction, consider $\frac{\partial^{2} v_{y}}{\partial x^{2}}$. In a configuration like in Figure 5, for an arbitrary $n$ the no-slip condition implies $v_{y, i-1, j, k}^{n}=0$. Hence, in such a configuration, we put

$$
\left.\frac{\partial^{2} v_{y}}{\partial x^{2}}\right|_{i, j, k} ^{n} \approx \frac{v_{y, i+1, j, k}^{n}-v_{y, i, j, k}^{n-1}-v_{y, i, j, k}^{n+1}}{h^{2}}
$$

In a situation shown in Figure 6, one obtains

$$
\left.\frac{\partial^{2} u_{y}}{\partial x^{2}}\right|_{i, j, k} ^{n} \approx \frac{-v_{y, i, j, k}^{n-1}-v_{y, i, j, k}^{n+1}}{h^{2}}
$$

If the stencil encounters a wall, as in Figure 7, then the no-slip boundary condition must be formulated at the midpoint marked by the triangle. The interface between the voxels $i-1, j, k$ and $i-1, j-1, k$ is inside the solid phase, so we use the center of this interface as a ghost node where $v_{y}$ is equal to $-v_{y i, j, k}^{n}$. Thus, the approximation of the second derivative of $v_{y}$ with respect to $x$ takes on the form:

$$
\left.\frac{\partial^{2} v_{y}}{\partial x^{2}}\right|_{i, j, k} ^{n} \approx \frac{v_{y, i+1, j, k}^{n}-v_{y, i, j, k}^{n}-v_{y, i, j, k}^{n+1}-v_{y, i, j, k}^{n-1}}{h^{2}}
$$

3.3.2. The boundary conditions at the boundary of the sample. To formulate the boundary conditions for the entire image of the sample, it is wrapped with a single layer of pore voxels. The boundary conditions 


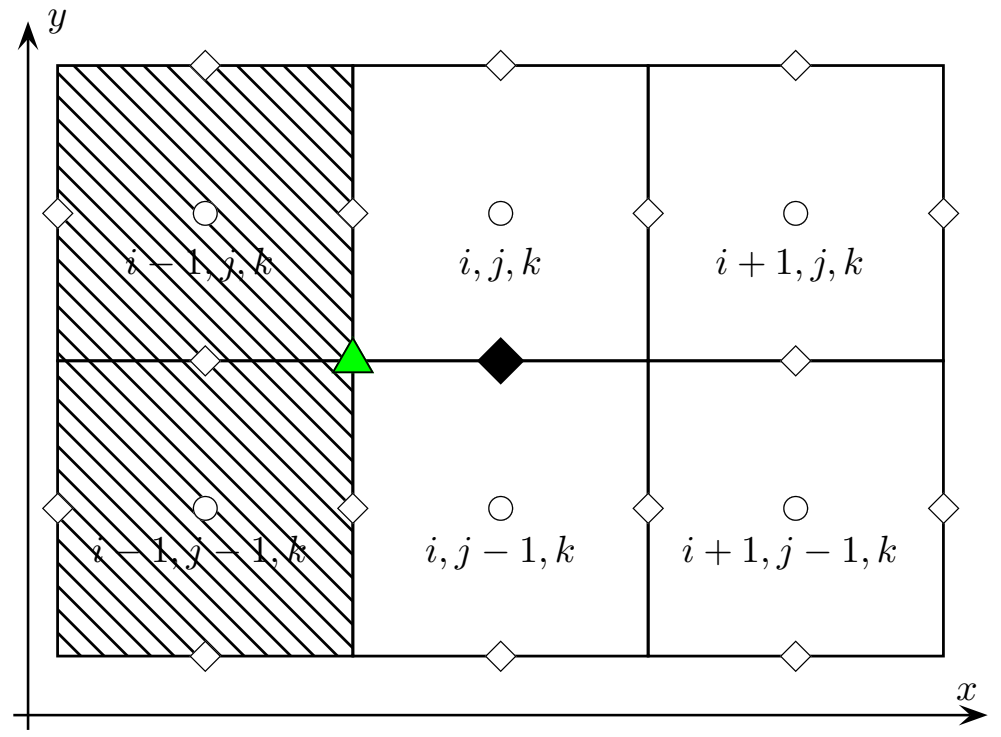

FiguRE 7. A stencil encountering a pore wall. The center of the stencil is labelled by a filled diamond, whereas the triangle marks the boundary between the solid wall and pore space.

are formulated at the planes passing through the centers of these wrapping voxels. The boundary nodes are shown as filled circles and filled diamonds in Figure 8.

To impose Dirichlet boundary conditions for the pressures and artifitial densities at the inlet and outlet, we merely assign the values at the respective voxels. For example, let the flow be in the $x$ direction. At all nodes $i, j, k$ with either $i=0$ or $i=N_{x}-1$ we assign the pressures to mimic the pressure gradient $(1,0,0)$. For example:

$$
\rho_{i, j, k}=1+\frac{\delta h\left(N_{x}-1-i\right)}{N_{x}-1}
$$

In fact, one can use Equation (20) as the initial condition for the densities over the entire pore space.

To formulate the boundary conditions for velocities, we have to add to the grid a single layer of ghost voxels at the downstream boundary in each direction. For example, in $x$ direction, next to the boundary voxels $N_{x}-1, j, k$, we define a double array of ghost velocity components $v_{x, j, k}^{\text {ghost }}$. The ghost arrays $v_{y, i, k}^{\text {ghost }}$ and $v_{z, i, j}^{\text {ghost }}$ are introduced in a similar manner.

Since the lateral velocity components are evaluated at the centers of the voxels faces, discretization of the zero-flow Dirichlet condition is 


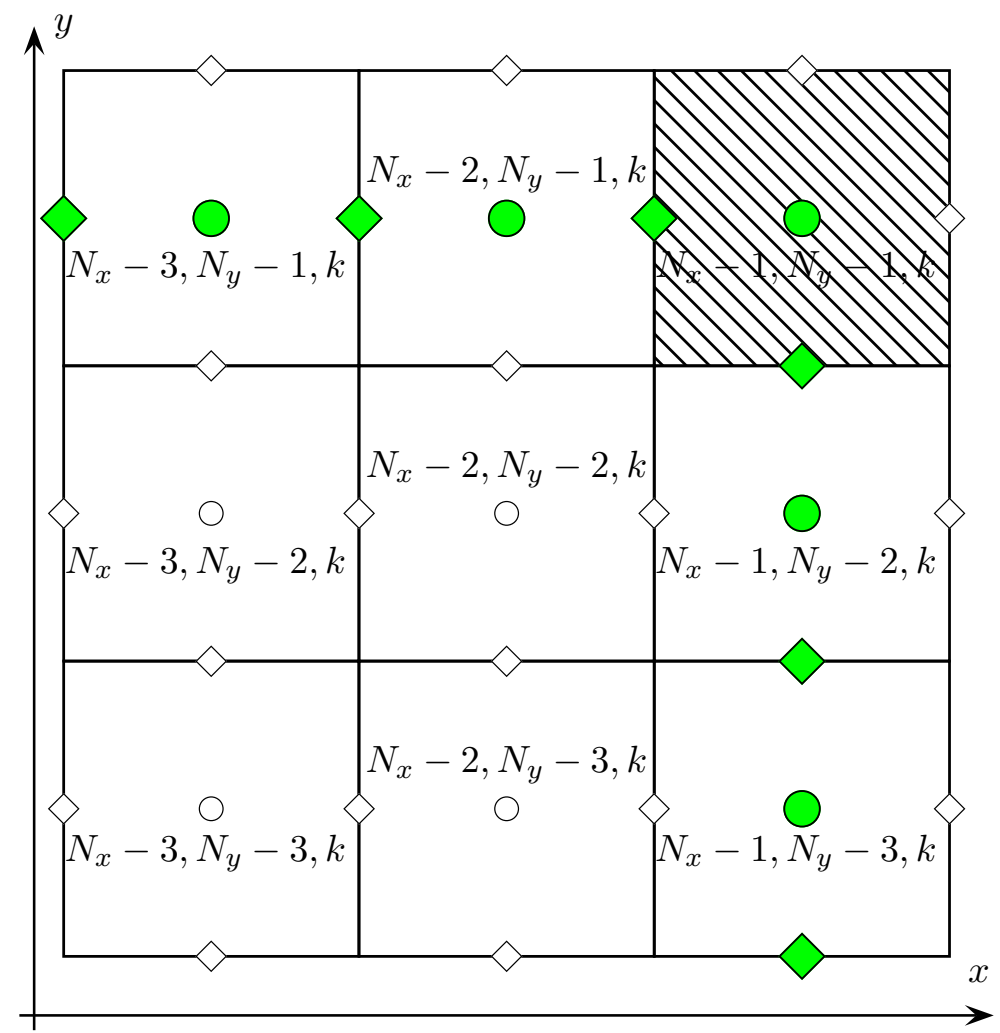

FiguRE 8. Discretization of the boundary conditions at the boundary of entire sample: filled circles and diamonds denote boundary nodes. The shaded corner voxel does not enter the discretized boundary conditions.

straightforward. For the component $v_{x}$, the central difference provides a second-order approximation of the normal derivative. That is, for example, for the zero Neumann boundary condition at the inlet voxels we put

for all $j, k$.

$$
v_{x, 0, j, k}=v_{x, 1, j, k}
$$

On each lateral boundary of the sample, two velocity components are evaluated at the boundary. For these components, discretization of the Dirichlet condition is straightforward. For the third component, a linear interpolation provides a second-order approximation. For example, for $v_{y}$ on the top boundary in Figure 8 one gets

$$
v_{y, i, N_{y}-1, k}+v_{y, i, k}^{\text {ghost }}=0
$$

where $v_{y, i, k}^{\text {ghost }}$ is the ghost velocity component defined above. 
Note that in the discretization described above the velocity components evaluated at the external boundaries of the vertex and edge voxels are not involved. The values of the artificial density $\rho$ at the corner nodes also are not involved in the computations.

3.4. Computational parameters. To choose values for the artificial compressibility, an iteration step $\tau$, and a stopping criterion, the algorithm has been applied to two problems admitting analytical solutions: Poiseuille flow in a pipe of square cross-section, and the Stokes problem of laminar flow around a solid sphere. We have chosen 10 voxel sizes as the dimensionless length unit, so that $h=0.1$. For each velocity component and the artificial density, the mean square relative increment was the measure of the increment at each iteration. For example, for the $x$ component of velocity, $v_{x}$, we evaluate

$$
D V_{x}^{n}=\frac{\sum_{i, j, k}\left|v_{x, i, j, k}^{n+1}-v_{x, i, j, k}^{n}\right|^{2}}{\sum_{i, j, k}\left(v_{x, i, j, k}^{n}\right)^{2}}
$$

The iteration continue until fulfilling the stopping criterion

$$
\max \left\{D V_{x}^{n}, D V_{y}^{n}, D V_{z}^{n}, D \rho^{n},\right\} \leq \varepsilon_{\text {tol }}
$$

The numerical computations for the Poiseuille flow showed stability of the algorithm for a wide range of artificial compressibility values. Figure 9 shows plots of exact and numerical profiles of velocity component $v_{x}=v_{x}(y)$ for the flow in $x$ direction for various cross-sections by planes orthogonal to the $z$ axis.

The Poiseuille flow example is very important in the context of simulation of the flow in the pore space. However, this example may not be very indicative when testing a numerical scheme for the convergence and stability. Indeed, the good convergence for the Poiseuille flow example should not be surprising. Although the computations are performed on a three-dimensional domain, the velocity component $v_{x}$ remains independent of $z$ in all iterations, and the other two components remain identically equal to zero. Thus, the inlet and outlet boundary conditions are satisfied automatically and the problem is actually solved in 2D. Moreover, due to the vanishing components, only one Dirichlet boundary-value problem for a Poisson equation is solved. In such a case, the stability and convergence of the DuFort-Frankel scheme has been demonstrated theoretically [35]. An additional testing of the algorithm on the Stokes problem of laminar flow around a solid sphere of radius $R$ in an infinite domain has shown that, actually, 


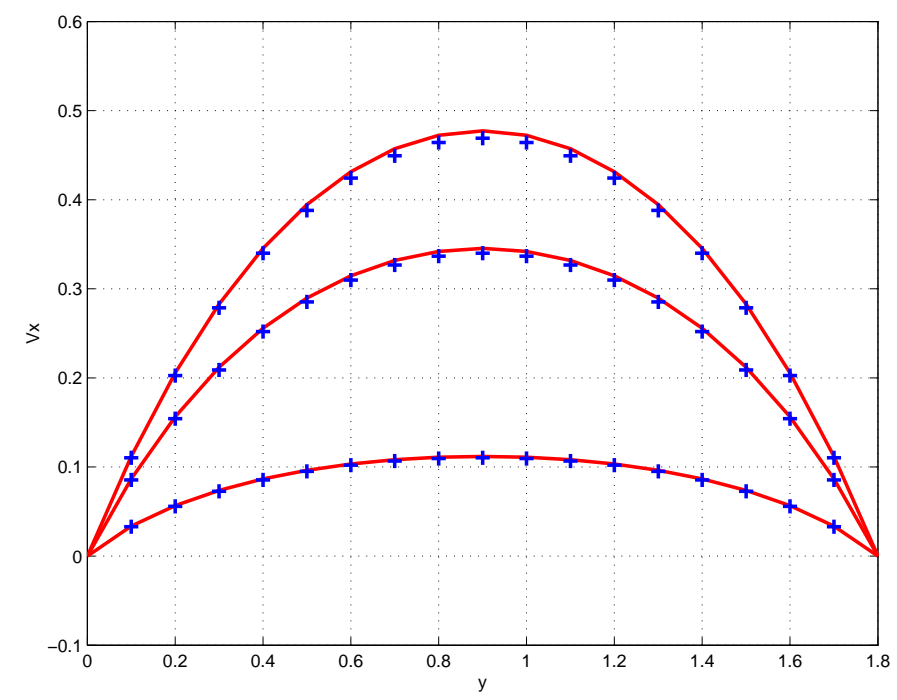

Figure 9. Comparison of computed and exact solutions for Poiseuille flow. The stopping criterion $\varepsilon_{\text {tol }}$ was set equal to $10^{-8}$.

the convergence and stability of the algorithm are both sensitive to the choice of the time step $\tau$. We have found that values near $\tau=10^{-3}$ were suitable both for the Poiseuille and Stokes problems.

On the surface of the sphere, the velocity satisfy no-slip boundary conditions, and it is equal to $\mathbf{v}_{\infty}$ at infinity. It is known, that the creeping-flow approximation adequately describes the flow at sufficiently small distances from the sphere surface [21]. The exact solution is given by equation

$$
\mathbf{v}(\mathbf{r})=\mathbf{v}_{\infty}-\frac{3 R}{4 r}\left(\mathbf{v}_{\infty}+\mathbf{n}\left(\mathbf{v}_{\infty} \cdot \mathbf{n}\right)\right)-\frac{R^{3}}{4 r^{3}}\left(\mathbf{v}_{\infty}-3 \mathbf{n}\left(\mathbf{v}_{\infty} \cdot \mathbf{n}\right)\right)
$$

Here $\mathbf{r}$ is the radius-vector from the center of the sphere, and $r$ is the distance from the center: $r=\|\mathbf{r}\|$. For the dimensionless pressure, one has

$$
p(\mathbf{r})=p_{\infty}-\frac{3 R}{2 r^{2}} \mathbf{u}_{\infty} \cdot \mathbf{n}
$$

where $p_{\infty}$ is the fluid pressure at infinity.

We evaluate numerically the flow near a sphere of the radius of 5 voxel widths. We embed the sphere in a $25 \times 25 \times 25$ voxel surrounding grid. At all outer boundaries of the entire domain, we impose Dirichlet conditions for the velocities using the trace of the exact solution (25). We found that an artificial compressibility near $\delta=0.05$ provided for the convergence and stability of the iterations. The same value 


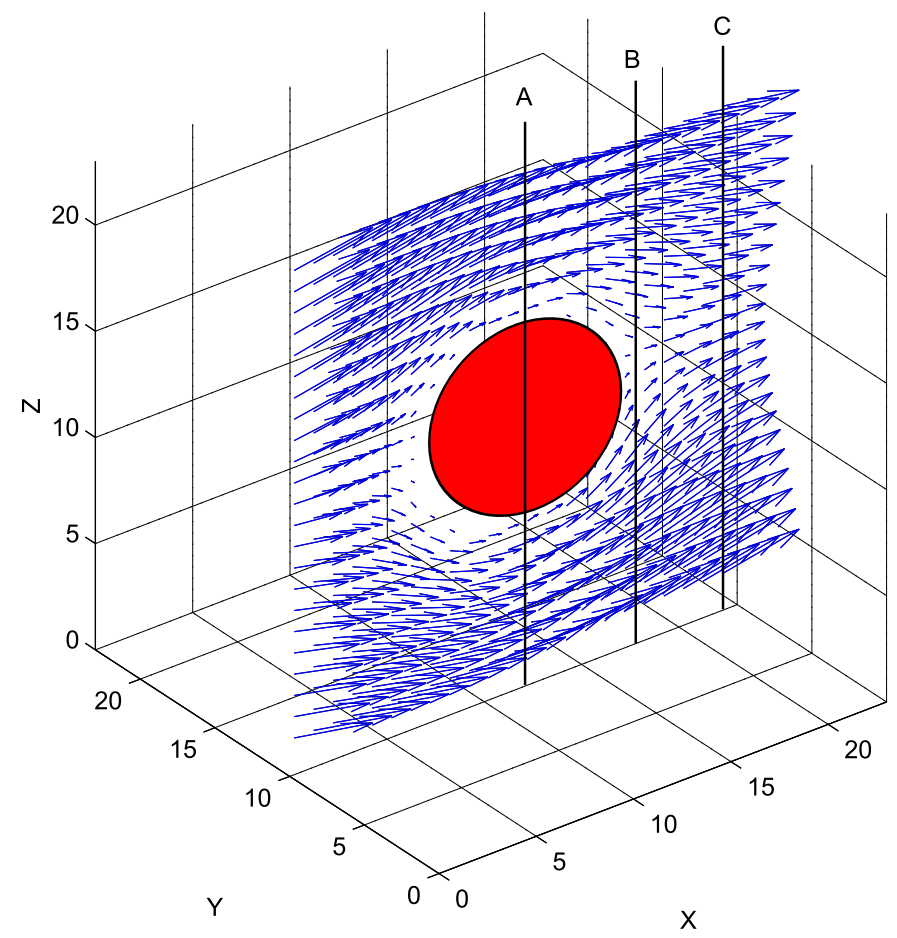

FIGURE 10. A central cross-section of the velocity field evaluated numerically.

worked well in the simulations of flow in a more complex geometry of the domain as well. A deviation from this value caused a loss of the convergence.

Figure 10 shows a cross-section of the numerically-evaluated vector field. The plot on Figure 11 shows three profiles of the $x$ component of the velocity, $v_{x}$, plotted versus $y$ for three different $x$. The bottom profile corresponds to the line A in Figure 10. The top profile is near the outlet boundary (line $\mathrm{C}$ ), and the one in the middle is at a midpoint between the sphere surface and the boundary, line B. To facilitate the comparison, the analytical and numerical solutions are evaluated at the voxel centers. The velocities obtained numerically are projected on the voxel centers by taking the arithmetic mean of the values at the opposite faces. The reason why neither the exact solution nor the numerical one attain zero at the sphere surface is that none of the voxel centers occurs exactly on the surface of the sphere. Figure 12 shows a similar comparison for the pressure profiles.

Even though the resemblance between the discretized sphere and an ideal spherical body is very approximate, Figure 14, the comparison 


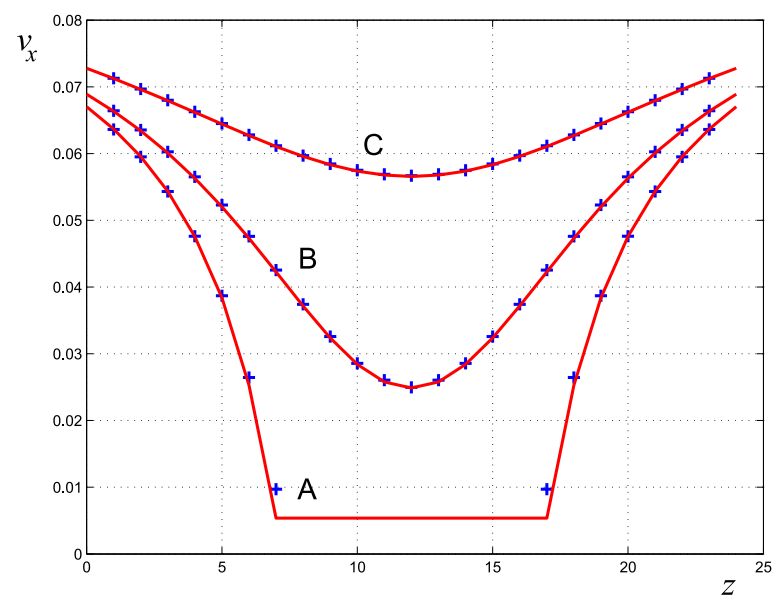

Figure 11. Tree velocity profiles corresponding to cross-sections A, B and C, Figure 10: a comparison between exact solution (Eq. (25)), shown as a solid line, and numerical solution, pluses, in dimensionless units.

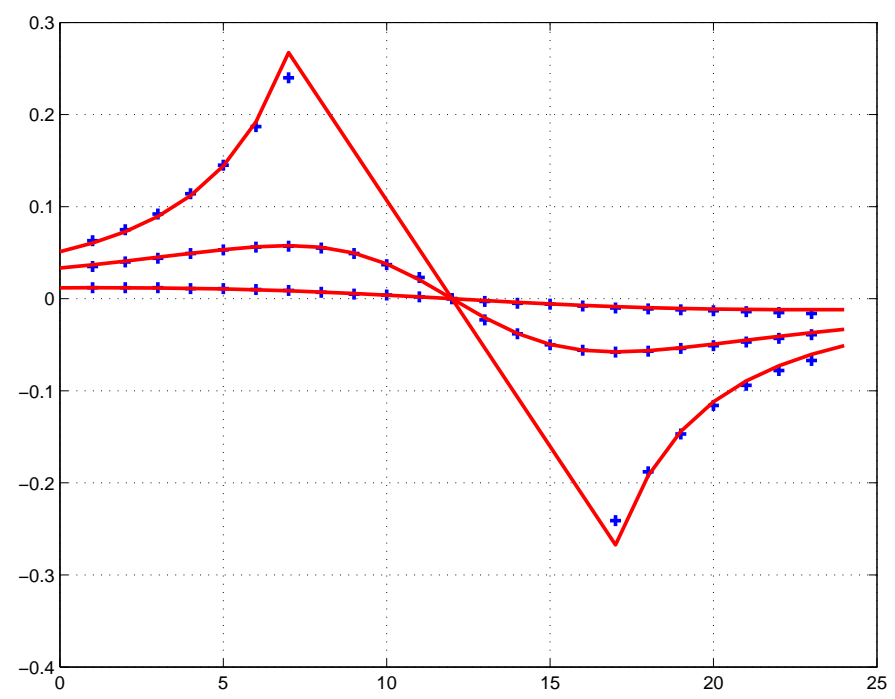

Figure 12. Tree pressure profiles: a comparison between exact solution (Eq. (25)), shown as a solid line, and numerical solution, pluses, in dimensionless units.

between the numerical and exact solutions is encouraging. One has to note though that this accuracy has been achieved by applying a rather stringent stopping criterion: $\varepsilon_{\mathrm{tol}}=10^{-8}$. Attempts of relaxation of 

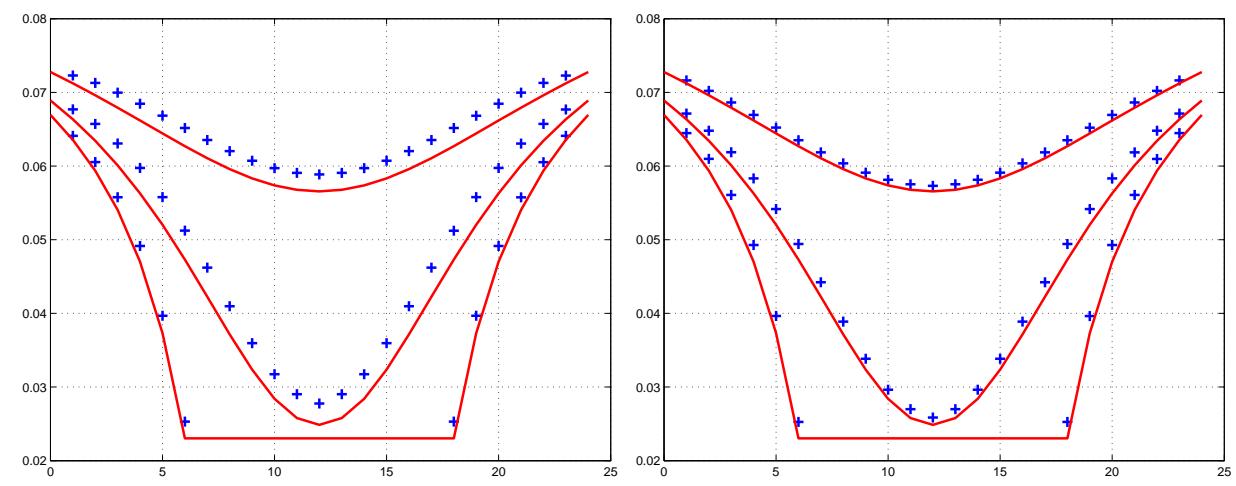

FiguRE 13. Velocity profiles shown in Figure 11 for relaxed stopping criterion: $\varepsilon_{t o l}=7.5 \times 10^{-7}$ on the left, and $\varepsilon_{t o l}=7.5 \times 10^{-8}$ on the right.
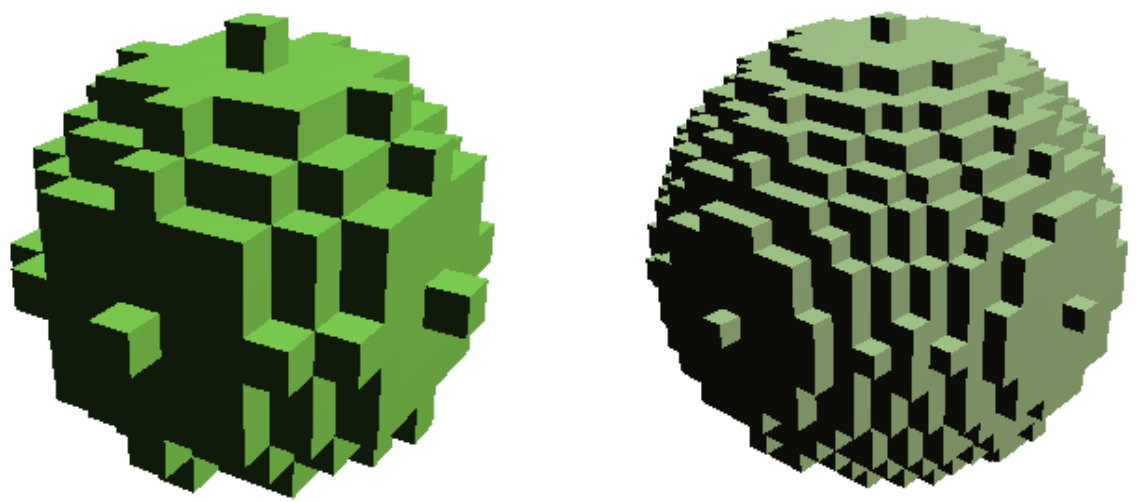

FIGURE 14. Discretized spheres of the radius of 5 voxel (on the left) and 10 (on the right) voxel units.

this criterion negatively affected the accuracy of the numerical solution, Figure 13. At the same time, further enhancement of the tolerance in the stopping criterion, $\varepsilon_{\text {tol }}=7.5 \times 10^{-10}$, resulted in no appreciable improvement of the accuracy.

The Darcy velocity is evaluated as the mean value of the fluid velocity over the entire grid. Figure 16 shows that the estimate of the component of the Darcy velocity in the downstream direction stabilizes after a sufficiently large number of iterations. The relative increments also stabilize near a value between $10^{-9}$ and $10^{-8}$, Figure 17.

A refinement of the discretization by reducing twice the voxel size has not significantly improved the accuracy of the numerical solution. The plots in Figure 15 compare the velocity profiles evaluated numerically for two grids and the exact solution. 


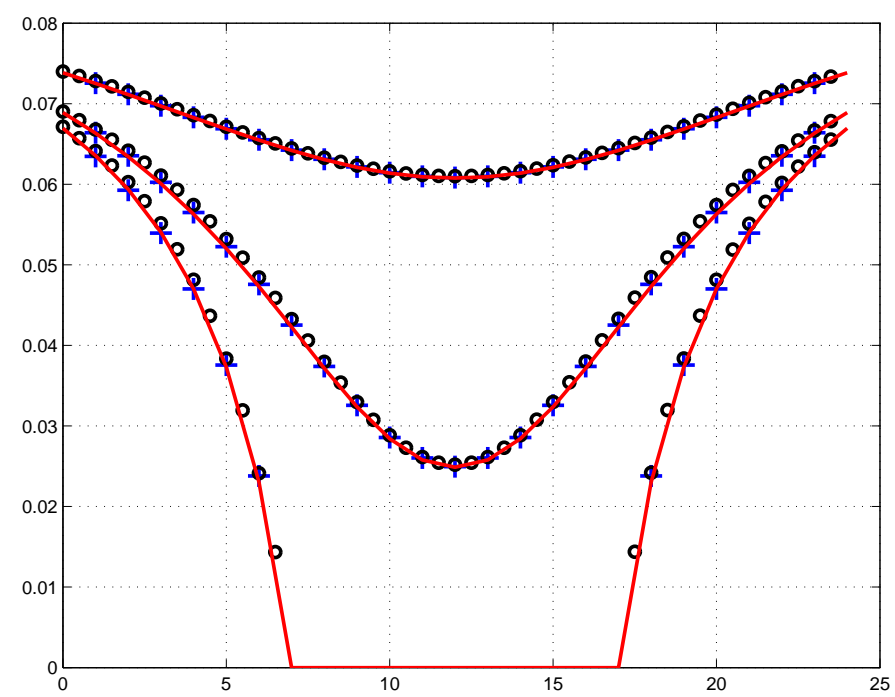

FIGURE 15. Velocity profiles evaluated numerically on coarse (pluses) and fine (circles) grids of voxels along with exact solution (solid line).

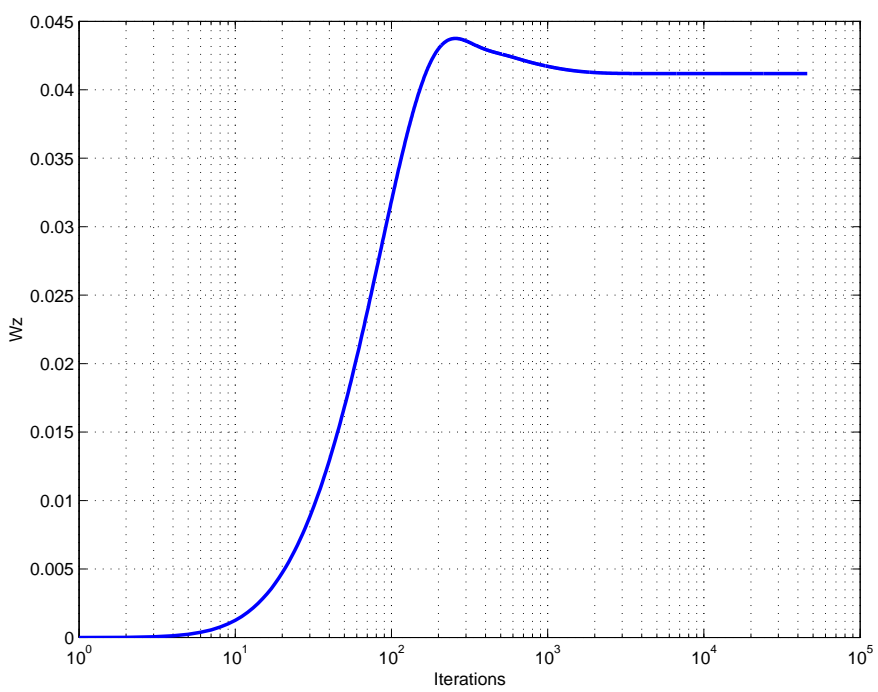

Figure 16. Stabilization of Darcy velocity estimate.

To summarize the results of testing the algorithms, two principal conclusions are due. First, a reasonable accuracy of the numerical computations requires a stringent stopping criterion. Second, the computations on a refined grid show that for the overall accuracy of the solution the convergence is more important than the voxel size. 


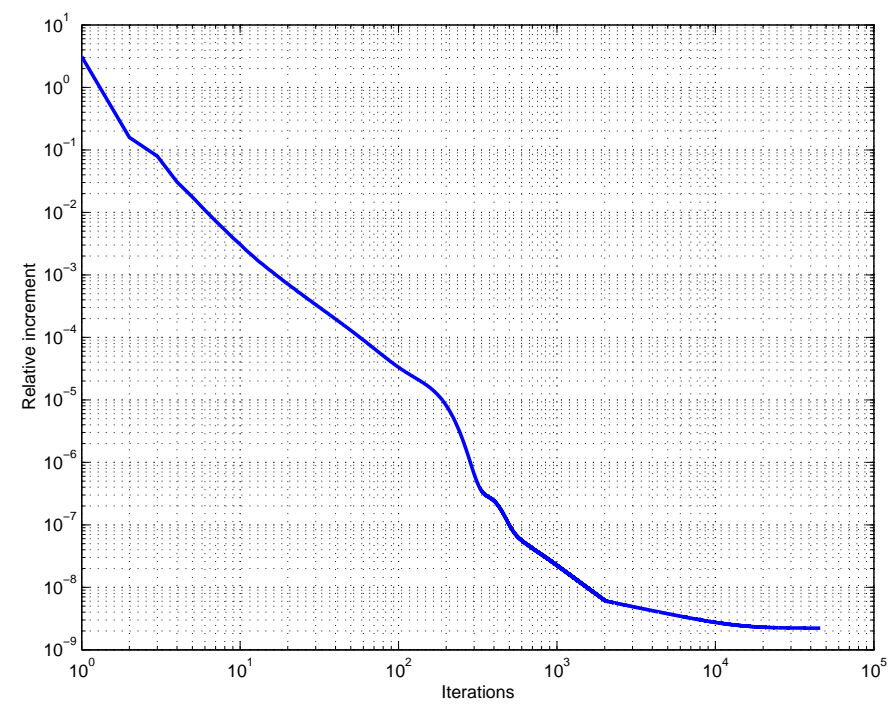

FiguRE 17. Stabilization of relative improvement near $10^{-9}$.

\section{Evaluation of the Permeability: PARtitioning of the IMAGE INTO LAYERS}

To estimate the permeability of a sample, we solve the Stokes equations on the pore space. Averaging the velocity field leads to an estimate of the Darcy velocity. The Dirichlet boundary conditions for the pressures at the inlet and outlet of the sample provide an estimate of the macroscopic pressure gradient. Finally, from the Darcy velocity and pressure gradient, one obtains an estimate of the permeability. We assume that the flow properties of the sample are isotropic. A repetition of the flow simulations in three coordinate direction, that is for three different choices of inlet and outlet boundaries, reduces the uncertainty of the estimates.

Meeting the severe convergence requirements discussed in Section 3.4 can be computationally demanding. It was impossible to attain the tolerance criterion on a large domain within a reasonable number of iterations. To reduce the dimensions of the computational domain, it is beneficial to split the entire sample into parts. Here we employ partitioning of the sample into layers, so that the lateral dimensions of each part significantly exceed the thickness. The simulations can be carried out on each part separately, and the the results can be combined by taking the harmonic mean of the permeability estimates for the individual layers. 
The computations within this study have been carried out on layers with the aspect ratios between the lateral dimensions and the thicknesses between 6 and 10. On each slice, the pressure boundary conditions are imposed on the larger faces of the sample, Figure 18. The permeability is evaluated three times by simulating flow in the three coordinate directions $x, y$, and $z$. To reduce the uncertainty, the average permeability estimate is used as the final output.Doubling the layer thicknesses produced very similar results. A more thorough analysis of the limiting admissible values of the aspect ratio, sample size, the number of layers, etc.would be a worthwhile task, but it is beyond the scope of this work.

The no-flow lateral boundary conditions can block the flow pathes which may leave or enter the sample. For example, if a rectangular sample is selected as in Figure 19, then flow simulations will show zero flow in the flow paths A, C, and D. If the image is sliced in the direction of the flow, the conductivity of these flow pathes will be non-zero at least for some slices. At the same time, the conductivity of a flow path of very complex geometry, like the one labelled by B, will be likely overestimated in the shadowed slice. Figure 19 is a cartoon illustration and cannot cover the full complexity of the 3D pore space geometry. However it indicates that the partitioning of the entire sample image into slices may result in an overestimate of the absolute permeability if, for example the layer crosses some flow path more than once. At the same time, the layers with large lateral dimensions reduce the impact of the blocking of the flow at the lateral boundaries. There is an uncertainty whether these two effects can compensate for one another.

4.1. Relative permeability curves. Two or multi-phase flows of immiscible fluids are described by the generalized Darcy's law. Based on the experiments by Wyckoff and Botset [27], the foundations of the theory of multi-phase flows in porous media have been developed by Muskat and Meres [26] and Leverett [22].

Consider, for example, simultaneous flow of water and oil. Since each fluid occupies only a part of the pore space, the permeability to this fluid is different from the absolute permeability of the rock. A dimensionless relative permeability is a correction factor, so that the Darcy's law for each fluid has the form:

$$
u_{o}=-\frac{k_{o} k}{\mu} \nabla p_{o}, \quad u_{w}=-\frac{k_{w} k}{\mu} \nabla p_{w}
$$

The relative volume of the pore space occupied by each fluid is its saturation. According to the classical theory, both relative permeability 


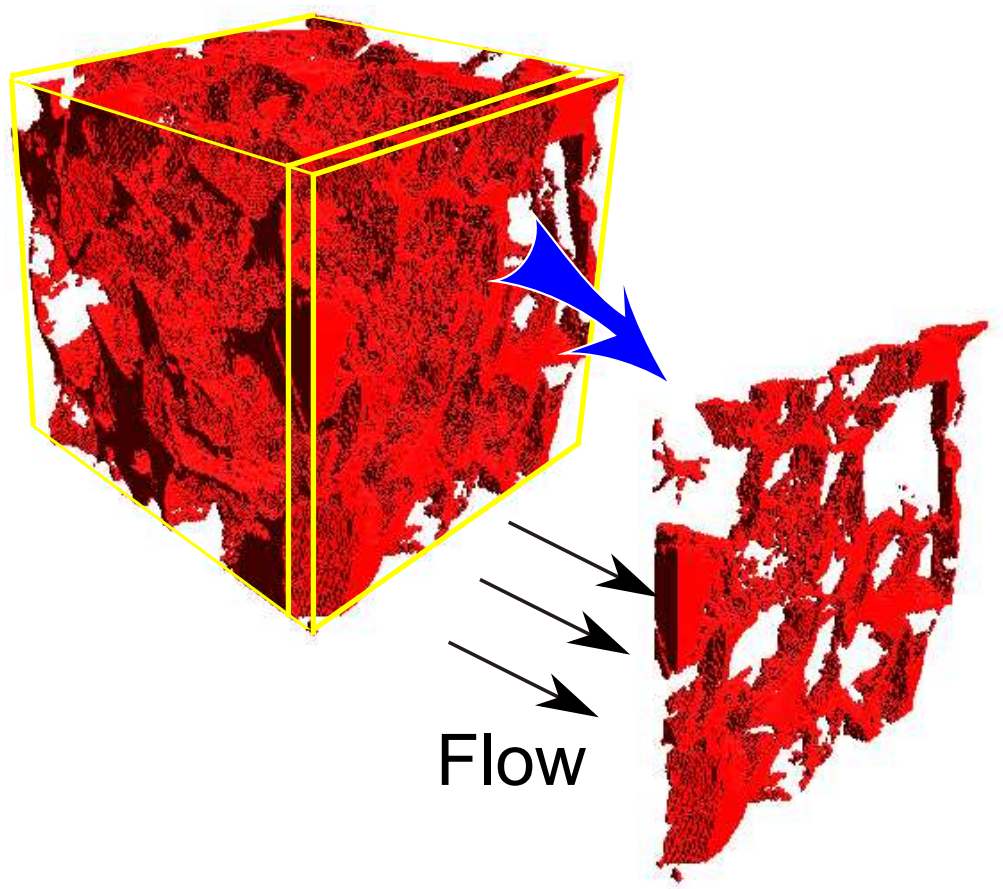

FiguRE 18. Partitioning of the image of the pore space into layers and solving flow equations for the flow orthogonal to the layer.

factors, $k_{o}$ and $k_{w}$, are functions of the fluid saturations, $S_{o}$ and $S_{w}$. Since the sum of both saturations is identically equal to one, the water saturations, $S_{w}$, completely characterizes the volumetric fluid distribution. Thus, one can write

$$
k_{o}=k_{o}(S), \quad k_{w}=k_{w}(S)
$$

where $S=S_{w}$.

One of the main assumptions of the classical theory of multi-phase flow in porous media is the local equilibrium of the fluid distribution enforced by the capillary pressure. In general, the picture is more complex and the flow rate, and the history of fluid displacement $[31,7]$ can have a significant impact on the geometry of the fluid-fluid interfaces.

In this work, we assume that local equilibrium is entirely defined by the capillary pressure. This assumption is suitable in a steady flow where the flow rate is small enough. In such a case, the distribution of the fluids in the pore space can be estimated using the method of maximal inscribed spheres (MIS). Briefly, the idea of the method is in assigning to each voxel the radius of the maximal sphere included in the pore space and covering this voxel. Once a three-dimensional table 


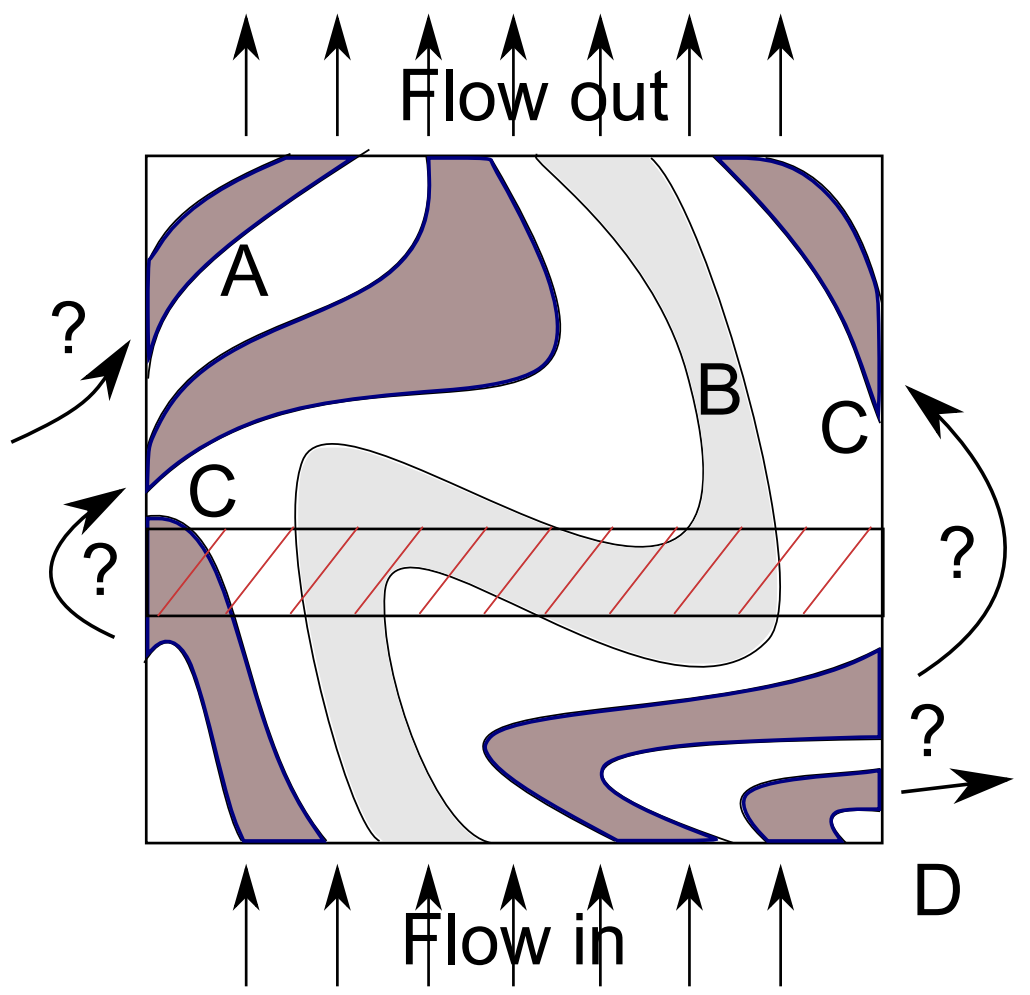

FiguRE 19. Uncertainties associated with modeling of the flow: Only path (B) is not blocked by the no-flow boundary condition. Some flow pathes contributing to the overall permeability may enter through a lateral boundary $(\mathrm{A})$, leave and return $(\mathrm{C})$, and just leave $(\mathrm{D})$. Partitioning of the image may lead to an overestimate of conductivity of channel (B) in some layers.

of such maximal radii is generated, one can simulate various invasion scenarios using cluster search algorithms. A more detailed description of the method is in $[38,41]$.

MIS method, in particular, involves evaluation of fluid distribution at a given capillary pressure. With a due scaling, it produces a reasonable approximation of the capillary pressure curve [43]. The calculated fluid distribution yields a list of the voxel which are deemed to be occupied by water or oil. The voxels occupied by one fluid can be considered as a stand-alone pore space. Thus, the permeability of this system of voxels scaled by the absolute permeability of the entire pore space is an approximation to the relative permeability factor. Counting the relative number of these voxels provides an estimate of the saturation. 
To summarize, the following procedure has been employed to evaluate the relative permeability curves numerically.

(1) Estimate the absolute permeability of the pore space:

- Run flow simulations in three directions;

- In each direction, partition the image into slices, estimate the permeability of each slice and take the harmonic mean;

- Take the average estimate for all three directions as the final output

(2) Calculate fluid distribution using MIS algorithm and evaluate the respective saturations:

- Evaluate the maximal inscribed radii;

- The total number of radii can be large, so choose a reasonable number for the flow simulations;

- For each chosen radius, compute the fluid distribution and saturation

(3) Evaluate relative permeabilities

- For each saturation chosen in the previous item, evaluate the permeability to each fluid;

- Evaluate the relative permeability by taking the ratio of the estimates of the permeabilities to each fluid to the estimate of the absolute permeability;

- Plot the relative permeabilities curves as functions of the saturation.

Figure 20 shows numerical results for CT images of a sandstone, and a number of experimental points obtained in coreflood laboratory experiments. A comparison can be only qualitative, since the images of the rock pore space have been acquired from similar, but not the same samples. One can see that the computed relative permeability curves, shown as solid curves with various markers on the plots, are in the same range as the experimental points. The variability of the computed relative permeabilities is similar to that of the data. Note that the scatter of the relative permeability estimated for the non-wetting fluid is larger than that for the wetting fluid. The greater likelihood of the non-wetting fluid loosing the connectivity can partially explain this observation.

In the simulations, we used the same digital data as in [41]. Most curves have been obtained using layers whose lateral dimensions are significantly larger than the thicknesses. The range of thicknesses was between 8 and 20 voxels. The images have a significant variability of the porosity [41]. Nevertheless, the permeability curves for the wetting 
fluid are aligned near the average curve. This indicates that the characteristic pore space geometry is a more important factor for the relative permeability estimates than the porosity. Figure 21 shows plots of the computed relative permeability curves for Finney pack [16]. All plotted data points have been obtained from flow simulations on one and the same domain of dimensions $160 \times 160 \times 160$ voxels. The different curves correspond to the different partitions of the image into layers of the thicknesses of $8,10,12$, and 16 voxels. The pair of solid thick curves is the arithmetic mean of all four results. The results are consistent, especially for the estimates of the relative permeability to the wetting fluid. However, the variability increases significantly for simulations on thicker layers. This variability mostly affects the estimates of the relative permeability to the non-wetting fluid, and is concentrated in the range of the "transient" saturations: between 40 and 75 percent. A refinement of the image resolution and more advanced computational algorithms for solving the Stokes equations apparently should extend the range of consistency of the computational results.

The fact that all simulations have been performed with no-slip boundary conditions also may introduce an uncertainty. Water is the wetting fluid. Therefore, the water-solid contact is rough and the length scale of this roughness is beyond the resolution of the image. This roughness may require more elaborate boundary conditions based on an asymptotic analysis. At the same time, the interfacial tension forces make the water-oil interface smooth, which cannot be captured by a digital image, where the boundaries are jagged unless they are aligned with the coordinate directions. At the fluid-fluid interface, such an artificial roughness of the surface may skew the momentum balance [34]. A further study can lead to a better understanding of the relative contribution of the roughness and discretization effects.

\section{Summary AND CONCLUSIONS}

Finite-differences solution of the incompressible creeping flow equations is a feasible approach to estimate flow properties of a porous medium from a three-dimensional computer tomography image of the pore space. In this work, we have studied numerical solution of the Stokes equations based on the method of artificial compressibility. The pore voxels of the digital image provide a natural mesh for discretization of the differential equations and boundary conditions. A two-layer Dufort-Frankel scheme on marker-and-cell staggered grid has been used in the numerical simulations. The computational parameters, including the coefficient of artificial compressibility, the magnitude of iteration 


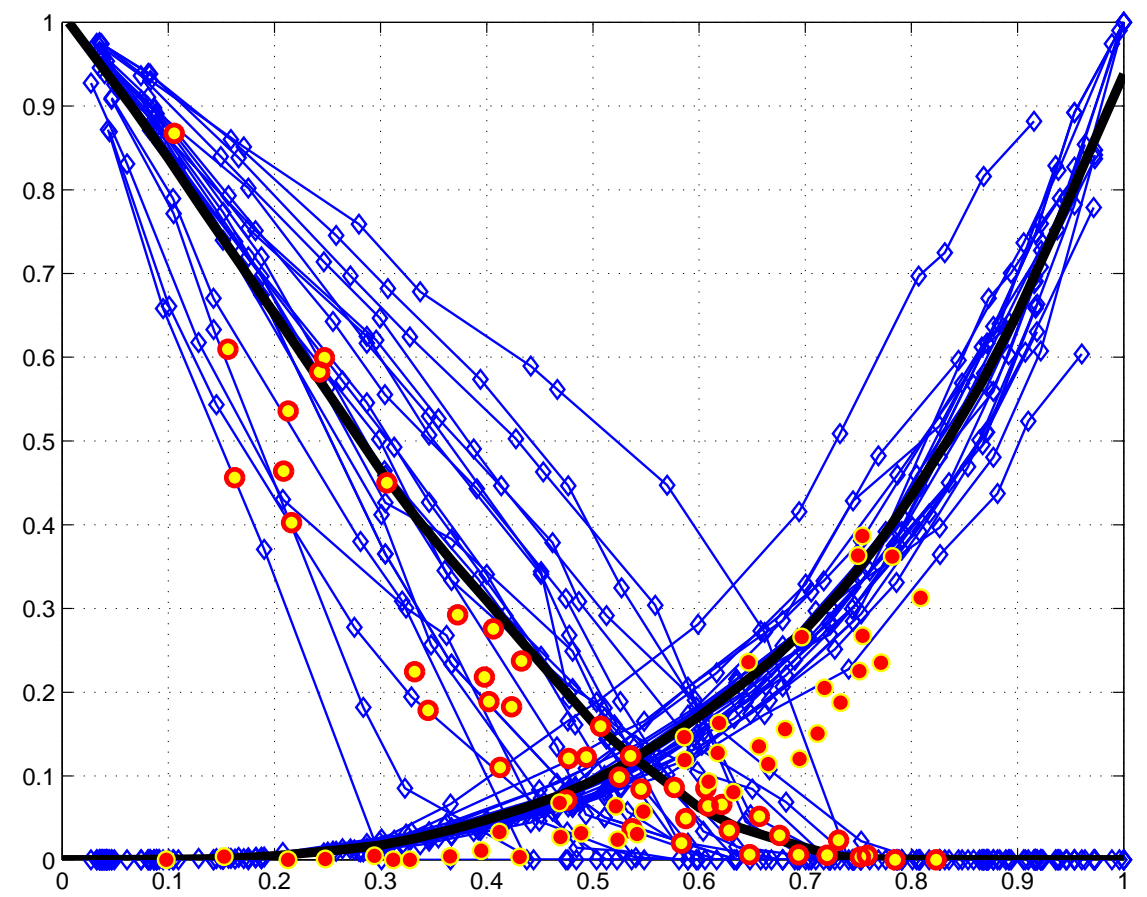

Figure 20. Computed relative permeability curves (solid lines with markers) and laboratory data (circles). The thick solid curves are average relative permeabilities.

The coreflood data are courtesy of Statoil.

step, and the stopping criterion, have been selected by trial and error. The criterion for stopping the iterations has been formulated in terms of the relative increment of the quadratic norm of the numerical solution. Numerical evaluation of the known analytical solutions, Poiseuille flow in a pipe of a rectangular cross-section and Stokes flow around a solid sphere, have demonstrated that the stopping criterion significantly affects the accuracy of the results. In fact, it turns out that a severe criterion is required to achieve a convergence suitable for dependable estimation of the permeability.

To reduce the computational intensity, the entire image of the porous sample is partitioned into layers. Prior to numerical simulations, the clusters of voxels, which are not connected to the inlet and outlet faces, are eliminated to reduce the size of the computational domain. Although this operation does not dramatically reduce the dimensions of the grid, it eliminates isolated stagnant-flow domains. The harmonic mean of the coefficients of permeability computed for the individual layers provides an estimate of the permeability of the entire sample. 


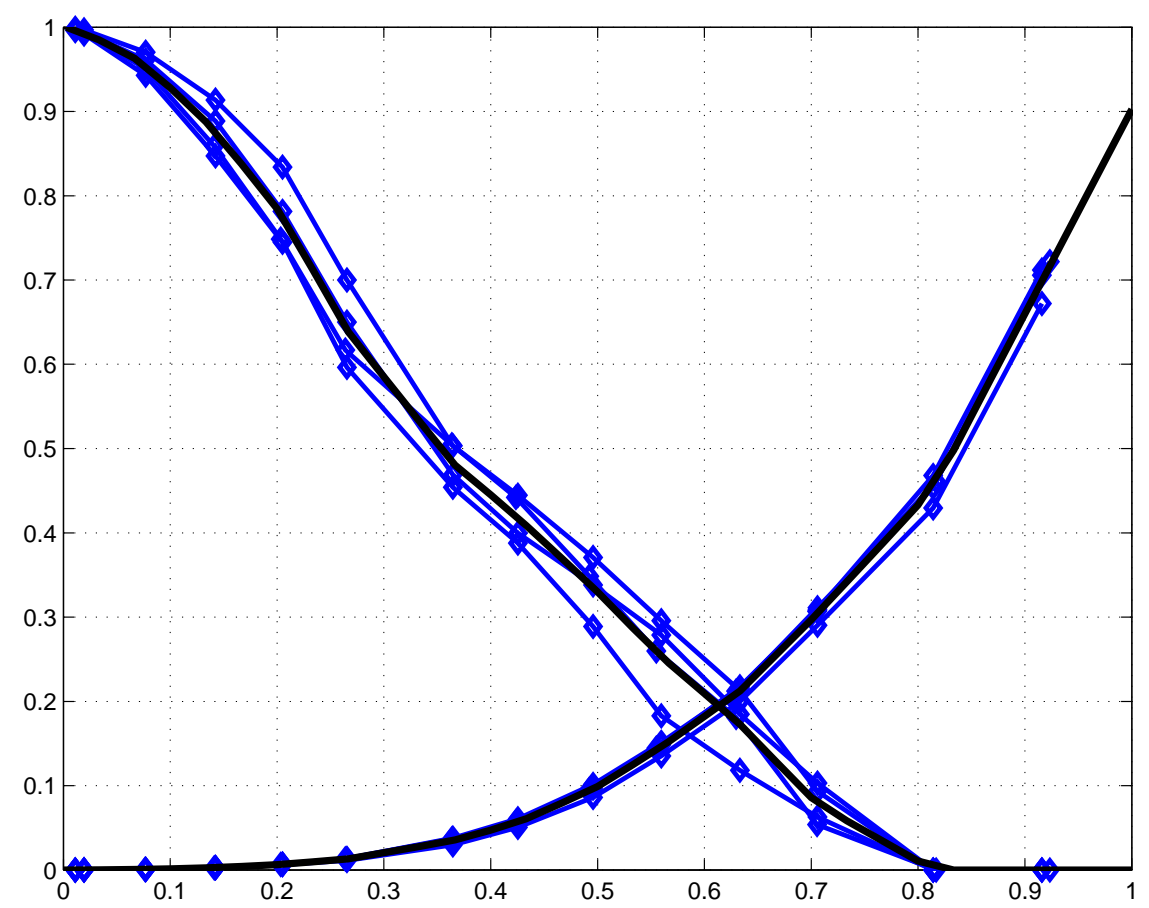

Figure 21. Computed relative permeability curves for Finney pack [16]. The thick solid curves are average Different curves correspond to four different thicknesses of the partitioning layers, Figure 18.

This approach makes possible to reduce the boundary effects introduced by the no-flow conditions at the lateral walls. At the same time, the partitioning into layers may lead to an overestimate of the permeability, for example, by accounting some flow channels multiple times. Evaluation of the permeability of a porous material from a small sample is always subject to a high degree of uncertainty. For an isotropic medium, averaging the results of flow simulations in three different coordinate directions can reduce this uncertainty.

The method of Maximal Inscribed Spheres calculates the capillaritydominated fluid distribution in the pore space of a water-wet rock. This distribution has been used to evaluate the sample permeability to each fluid at a given saturation. The relative permeability is defined as the dimensionless ratio of the phase permeability to the absolute permeability of the medium. The permeability to each phase at a given saturation has been estimated using the same algorithm as for the evaluation of the absolute permeability. The computations performed 
on 3D micron-scale resolution images of a sandstone produced relative permeability curves reproducing the experimental data amazingly well.

The approach discussed here is a promising tool for analysis of the petrophysical properties of natural rocks and other types of porous media. A direct analysis of the image of the pore space bypasses the challenges of network extraction unavoidable in the pore-network analysis. The proposed method can be applied to evaluate the impact of pore space geometry modifications on the rock flow properties. Such modifications can come from mechanical or thermal deformations, damage, formation or dissociation of hydrates, mineral deposition or dissolution. Exact numbers are subject to great uncertainty, both in numerical and laboratory experiments. Numerical simulations can generate trends and, therefore, provide useful insights for optimization of hydrocarbon recovery technologies, or efficient environmental remediation. The robustness of the algorithms makes the method suitable for routine applications, requiring minimum human interference. The current trends in the price of the computer time and data storage makes this approach even more attractive.

\section{ACKNOWLEDGMENTS}

This work has been performed at Lawrence Berkeley National Laboratory (LBNL) of the U.S. Department of Energy under Contract No. DE-AC02-05CH11231 and the University of California, Berkeley. The first author acknowledges support from the UC Oil Consortium and from the U.S. Department of Energy's Assistant Secretary for Coal through the Zero Emission Research and Technology Program under the U.S. Department of Energy Contract No. DEAC02-05CH11231 to LBNL. The authors are thankful to Dr. Andrea Cortis and Dr. Jonathan Ajo-Franklin of the Earth Sciences Division for reviewing the manuscript and useful suggestions.

\section{REFERENCES}

1. P. M. Adler, Fractal porous media III: Transversal Stokes flow through random and Sierpinski carpets, Transport in Porous Media 3 (1988), no. 2, 185-198.

2. A. Al-Futaisi and T. W. Patzek, Impact of wettability on two-phase flow characteristics of sedimentary rock: Quasi-static model, Water Resources Research 39 (2003), no. 2, 10421055.

3. F. M. Auzerais, J. Dunsmuir, B. B. Ferreol, N. Martys, J. Olson, T. S. Ramakrishnan, D. H. Rothman, and L. M. Schwartz, Transport in sandstone: A study based on three dimensional microtomography, Geophysycal Research Letters 23 (1996), 705-708. 
4. F. G. Avkhadiev and Anvar R. Kacimov, Analytical solutions and estimates for microlevel flows, Journal of Porous Media 8 (2005), no. 2, 125-148.

5. S. Bakke and P. E. Øren, 3-D pore-scale modeling of sandstones and flow simulations in the pore networks, SPE Journal 2 (1997), 136-149.

6. Sally M. Benson, L. Tomutsa, D. Silin, T. Kneafsey, and L. Miljkovic, Core scale and pore scale studies of carbon dioxide migration in saline formations., 8th International Conference on Greenhouse Gas Control Technologies. Trondheim, Norway, 19-22 June 2006., 2006.

7. M. J. Blunt, Flow in porous media - pore-network models and multiphase flow, Current Opinion in Colloid \& Interface Science 6 (2001), no. 3, 197-207.

8. M. J. Blunt and P. King, Relative permeabilities from two- and threedimensional pore-scale metwork modeling, Transport in Porous Media 6 (1991), 407-433.

9. M. J. Blunt and H. Sher, Pore network modeling of wetting, Physical Review E 52 (1995), no. December, 6387.

10. A. J. Chorin, A numerical method for solving incompressible viscous flow problems, Journal of Computational Physics 2 (1967), 12-26.

11. _ Numerical solution of the Navier-Stokes equations, Math. Comp. 22 (1968), 745-762.

12. V. M. Entov, The micromechanics of flow in porous media, Soviet Academy Izvestia. Mechanics of Gas and Fluids (1992), no. 6, 90-102.

13. I. Fatt, The network model of porous media. 1. Capillary pressure characteristics, Trans. AIME 207 (1956), no. 7, 144-159.

14. _ The network model of porous media. 2. Dynamic properties of a single size tube network, Trans. AIME 207 (1956), no. 7, 160-163.

15. __ The network model of porous media. 3. Dynamic propertries of networks with tube radius distribution, Trans. AIME 207 (1956), no. 7, 164-181.

16. J. L. Finney, Random packings and the structure of simple liquids. I. The geometry of random close packing, Proceedings of the Royal Society of London. Series A 319 (1970), no. 1539, 479-493.

17. Olav van Genabeek and Rothman Daniel H., Macroscopic manifestations of microscopic flows through porous media, Annual Review of Earth and Planetary Sciences 24 (1996), 63-87.

18. F.H. Harlow and J.E. Welsh, Numerical calculation of time dependent viscous incommpressible flow with free surface, Physics and Fluids 8 (1965), 2182-2189.

19. Xiaoyi He, Gary D. Doolen, and T. Clark, Comparison of the Lattice Boltzmann method and the artificial compressibility method for Navier-Stokes equations, Journal of Computational Physics 179 (2002), no. 2, 439-451.

20. G. Jin, T.W. Patzek, and D. B. Silin, Direct prediction of the absolute permeability of unconsolidated and consolidated reservoir rock. SPE 90084, $2003 \mathrm{SPE}$ Annual Technical Conference and Exhibition (Houston, Texas, U.S.A.), SPE, 2004.

21. L. D. Landau and E. M. Lifschitz, Fluid mechanics, Series in advanced physics, vol. 6, Addison-Wesley, Reading, MA, 1959.

22. M. C. Leverett, Flow of oil-water mixtures through unconsolidated sands, Trans. AIME 132 (1939), 381-401. 
23. C. Manwart, U. Aaltosalmi, A. Koponen, R. Hilfer, and J. Timonen, LatticeBoltzmann and finite-difference simulations for the three-dimensional porous media, Physical Review E 66 (2002), 016702 1-11.

24. C. Manwart and R. Hilfer, Numetrical simulations of creeping fluid flow in reconstruction models of porous media, Physica A 314 (2002), 706-713.

25. N. S. Martys and H. Chen, Simulation of multicomponent fluids in complex three-dimensional geometries by the lattice Boltzmann method, Physical Review E 53 (1996), 743.

26. M. Muskat and M. W. Meres, The flow of hetereogeneous fluids through porous media, 7 (1936), 346-363.

27. M. Muskat and M.W. Meres, The flow of gas-liquid mixtures through unconsolidated sands, 7 (1936), 325-345.

28. David R. Noble, Shiyi Chen, John G. Georgiadis, and Richard O. Buckius, A consistent hydrodynamic boundary condition for the lattice boltzmann method, Physics of Fluids 7 (1995), no. 1, 203-209.

29. R. M. O'Connor and J. T. Fredrich, Microscale flow modelling in geologic materials, Physics and Chemistry of the Earth, Part A: Solid Earth and Geodesy 24 (1999), no. 7, 611-616.

30. P. E. Øren, S. Bakke, and O. J. Arntzen, Extending predictive capabilities to network models, SPE Journal (1998), no. December, 324-336.

31. T. W. Patzek, Verification of a complete pore network simulator of drainage and imbibition, SPE Journal 6 (2001), no. 2, 144-156.

32. T.W. Patzek and J. G. Kristensen, Shape factor and hydraulic conductance in noncircular capillaries: II. Two-phase creeping flow, Journal of Colloid and Interface Science 236 (2001), 305-317.

33. T. W. Patzek and D. B. Silin, Shape factor and hydraulic conductance in noncircular capillaries: I. One-phase creeping flow, Journal of Colloid and Interface Science 236 (2001), 295-304.

34. T. C. Ransohoff and C. J. Radke, Laminar flow of a wetting liquid along corners of a predominantly gas-occupied noncircular pore, Journal of Colloid and Interface Science 121 (1998), 392-401.

35. A. A. Samarskii, The theory of difference schemes, M. Decker, New York, 2001.

36. L. M. Schwartz, N. Martys, D. P. Bentz, E. J. Garboczi, and S. Torquato, Cross-property relations and permeability estimation in model porous media, Physical Review E 48 (1993), no. 6, 4584 - 4591.

37. D. Silin and T. Patzek, Pore space morphology analysis using maximal inscribed spheres, Physica A Statistical Mechanics and its Applications 371 (2006), 336360 .

38. D. B. Silin, G. Jin, and T.W. Patzek, Robust determination of the pore-space morphology in sedimentary rocks. SPE 84296, 2003 SPE Annual Technical Conference and Exhibition (Denver, Colorado, U.S.A.), SPE, 2003.

39. D. B. Silin and T. W. Patzek, An object-oriented cluster search algorithm, Tech. Report LBNL-51599, Lawrence Berkeley National Laboratory, 2003.

40. _ Object-oriented cluster search for an arbitrary pore network, Tech. Report LBNL-51599, Lawrence Berkeley National Laboratory, Berkeley, CA, January 2003.

41. __ Pore space morphology analysis using maximal inscribed spheres, Physica A 371 (2006), 336-360. 
42. Dmitry B. Silin, Guodong Jin, and Tad W. Patzek, Robust deteremination of the pore-space morphology in sedimentary rocks, Journal of Petroleum Technology (2004), 69-70.

43. Liviu Tomutsa, Dmitriy Silin, and Velimir Radmilovic, Analysis of chalk petrophysical properties by means of submicron-scale pore imaging and modeling, SPE Reservoir Evaluation and Engineering 10 (2007), no. 3, 285-293.

Lawrence Berkeley National Laboratory, 1 Cyclotron Road, MS 90-1116, BERKELEy, CA 94720, USA

E-mail address: DSilin@lbl.gov

Chairman, the Department of Petroleum and Geosystems Engineering The University of TeXas At Austin CPE 2.502 Austin, TX 78712

E-mail address: patzek@mail.utexas.edu 